\title{
Speed of Ultrasound and Internal Pressure of Propanediol and Butanediol Isomers under Elevated Pressures
}

\author{
E. ZorȩBski, M. Piotrowska And M. Dzida \\ Institute of Chemistry, University of Silesia, Szkolna 9, 40-006 Katowice, Poland \\ Speed of ultrasound and internal pressure of 1,2-propanediol, \\ 1,3-propanediol, 1,2-butanediol, and 1,3-butanediol at the temperatures \\ ranging from 293.15 to $318.15 \mathrm{~K}$ and pressures up to $101 \mathrm{MPa}$ are analyzed \\ and discussed in terms of molecular structure and ability to form inter- and \\ intramolecular hydrogen bonds.
}

PACS numbers: 82.30.Rs, 62.50.-p

\section{Introduction}

The phase speed of an ultrasound wave depends on the elastic properties of the material. It is a significant source of information on the molecular structure of the liquid and on physical and chemical processes occurring in the liquid phase. Speed of ultrasound measurements carried out as functions of temperature and pressure enable also the determination of $p \rho T$ relationships. Moreover, the measurements of the speed of ultrasound permit the investigation of very subtle effects such as, for example, stacking effect [1] or the effect of the position of $\mathrm{OH}$ groups in diol isomers on the physicochemical and thermodynamic properties of the pure compounds or their mixtures [2]. However, it requires measurements of adequate high accuracy and precision [3-6].

One of the convenient methods for the determination of the internal pressure is the acoustic one, i.e. the use of speed of ultrasound in conjunction with the density, isobaric heat capacity, and isobaric expansibility. The starting point is the combination of the low-frequency speed of ultrasound and density, which yields the isentropic compressibility (the Newton-Laplace equation). Then the latter may be combined with the isobaric expansibility and the isobaric heat capacity to yield the isothermal compressibility and finally the internal pressure (more details can be found elsewhere [7-11]). The so-called direct methods of determination of internal pressure are very inconvenient. Therefore, these methods are rather rarely used. 
The internal pressure $P_{\text {int }}\left(P_{\text {int }}=(\partial U / \delta V)_{T}, U\right.$ is the internal energy) provides a useful basis for a better understanding of the structure and nature of intermolecular interactions in the liquid state. Simultaneously, a literature survey shows that such data are generally very scarce.

The well-known relationships for the calculation of the $P_{\text {int }}$, is given below

$$
P_{\text {int }}=\alpha_{p} T \kappa_{T}^{-1}-p
$$

where $\alpha_{p}$. is isobaric expansibility and $\kappa_{T}$ is isothermal compressibility.

It should be also noted that, first of all, errors in the determination of the $\alpha_{p}$ values lead to relatively large errors in $P_{\text {int }}$. In other words, from the quantities determined indirectly by the acoustic method, $P_{\text {int }}$ is most of all sensitive to errors in the $\alpha_{p}$ values. Therefore, reliable experimental values of $\alpha_{p}$ are desirable in such calculations.

In this work, the effects of pressure and temperature on the speed of ultrasound and internal pressure for 1,2-propanediol, 1,3-propanediol, 1,2-butanediol and 1,3-butanediol are analyzed and discussed. The data are taken from our previous papers $[7,8]$. These data covered temperatures ranging from 293.15 to $318.15 \mathrm{~K}$ and pressures up to $101 \mathrm{MPa}$. The uncertainty of the mentioned above $c$ and $P_{\text {int }}$ data have been estimated roughly to be about $\pm 1 \mathrm{~m} \mathrm{~s}^{-1}$ and $\pm 1 \%[7,8]$, respectively.

\section{Results and discussion}

The data necessary for the calculations were taken from the previous reports $[7,8]$. It appears that for a given pressure, the speed of ultrasound decreases almost linearly with increasing temperature, while at a given temperature, the speed of ultrasound increases non-linearly with increasing pressure. Moreover, with increasing pressure its effect on the speed of ultrasound is smaller. The values of $(\partial c / \partial p)_{T}$ at atmospheric pressure varied slightly, but converged to nearly a single value at pressures higher than $100 \mathrm{MPa}$. Generally, both temperature and pressure dependences are for all four diols typical of liquids.

In the case of $P_{\text {int }}$ results are given in graphic form in Figs. 1-4. The presented isotherms of $P_{\text {int }}(p)$ are obtained by fitting to a polynomial of the form

$$
P_{\text {int }}=\sum_{i=1}^{m} a_{i}(p / 100)^{i-1},
$$

where $a_{i}$ are coefficients obtained by a least squares method and $m$, the upper limit of the summation, is equal to three or four. The coefficients of the isotherms with evident maximum are summarized (together with the standard deviations $\delta$ ) in Table.

The internal pressure is related to the isothermal change of entropy per unit volume and can be discussed in terms of order in liquids resulting from isothermal expansion. This is very interesting property that can be calculated by the acoustic method. Calculated in this way the internal pressures of 1,2-butanediol and 


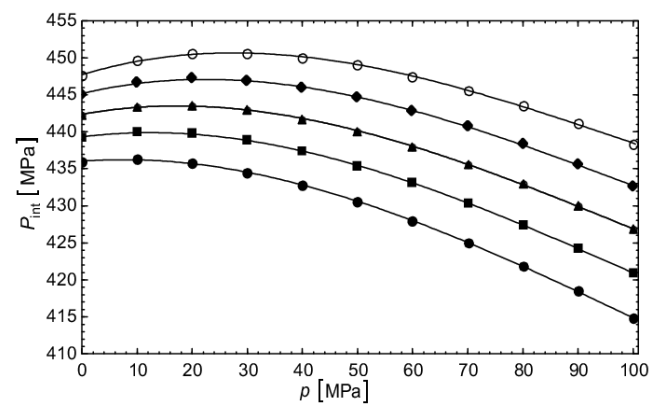

Fig. 1. Internal pressure $P_{\text {int }}$ as a function of pressure $p$ for 1,2-propanediol at the temperatures • $293.15 \mathrm{~K}$; $298.15 \mathrm{~K} ; \boldsymbol{\Delta} 303.15 \mathrm{~K}$; $\quad 308.15 \mathrm{~K}$; ○ 313.15 K. Lines Eq. (2).

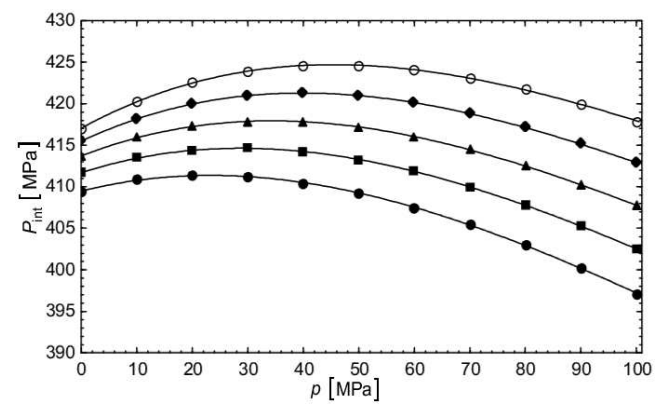

Fig. 2. As in Fig. 1, but for 1,2-butanediol.

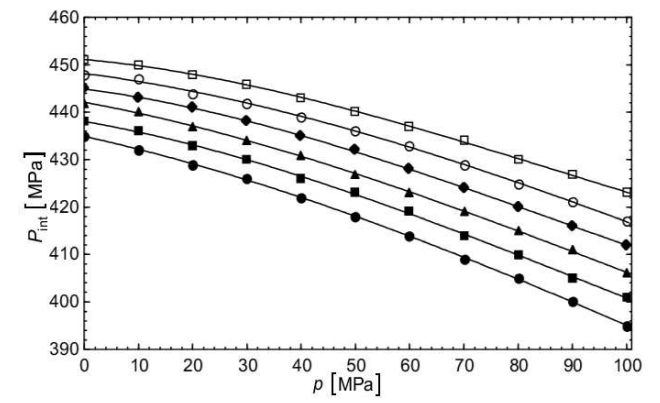

Fig. 3. Internal pressure $P_{\text {int }}$ as a function of pressure $p$ for 1,3-propanediol at the temperatures • $293.15 \mathrm{~K}$; $298.15 \mathrm{~K} ; \boldsymbol{\Delta} 303.15 \mathrm{~K} ; \bullet 308.15 \mathrm{~K} ; \circ 313.15 \mathrm{~K} ; \square 318.15 \mathrm{~K}$. Lines - Eq. (2).

1,2-propanediol, as function of pressure show maximum for all isotherms in the investigated temperature range (Table and Figs. 1, 2). In contrast, the internal pressure of 1,3-butanediol decreases monotonically with increasing pressure with the exception of the $T=318.15$ and $313.15 \mathrm{~K}$ isotherms. However, in this last case the maximum is very, very flattish (in the range of declared uncertainty of 


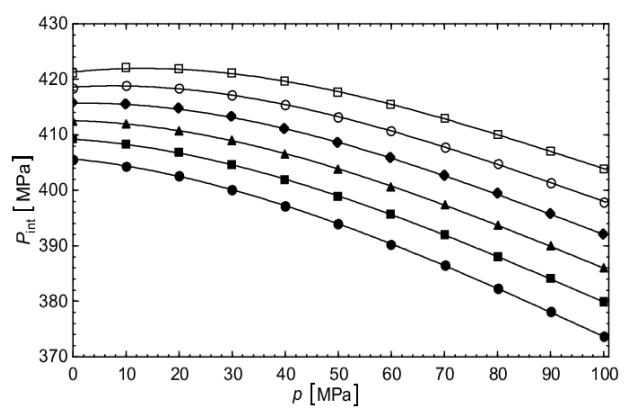

Fig. 4. As in Fig. 3, but for 1,3-butanediol.

TABLE

Coefficients $a_{i}$ of Eq. (2) together with standard deviation $\delta$ and estimated $p_{\max }$ at which $\left(\partial P_{\text {int }} / \partial p\right)_{T}=0$.

\begin{tabular}{c|c|c|c|c|c|c}
\hline \hline$T[\mathrm{~K}]$ & $a_{1}$ & $a_{2}$ & $a_{3}$ & $a_{4}$ & $p_{\max }[\mathrm{MPa}]$ & $\delta[\mathrm{MPa}]$ \\
\hline 293.15 & 436.03 & 5.648 & -39.06 & 12.25 & 7.5 & 0.1 \\
298.15 & 439.32 & 9.483 & -40.88 & 13.01 & 12.3 & 0.1 \\
303.15 & 442.36 & 13.58 & -42.91 & 13.85 & 17.3 & 0.1 \\
308.15 & 445.16 & 17.96 & -45.19 & 14.80 & 22.3 & 0.1 \\
313.15 & 447.72 & 22.61 & -47.71 & 15.86 & 27.5 & 0.1 \\
\hline \multicolumn{7}{c}{1,2 -butanediol ${ }^{a}$} \\
\hline 293.15 & 409.47 & 16.88 & -40.10 & 10.95 & 23.3 & 0.1 \\
298.15 & 411.70 & 21.37 & -42.19 & 11.66 & 28.7 & 0.1 \\
303.15 & 413.70 & 26.15 & -44.56 & 12.47 & 34.3 & 0.1 \\
308.15 & 415.48 & 31.30 & -47.34 & 13.47 & 39.8 & 0.1 \\
313.15 & 417.04 & 36.73 & -50.36 & 14.54 & 45.4 & 0.1 \\
\hline \multicolumn{7}{c}{1,3 -butanediol ${ }^{a}$} \\
\hline 313.15 & 415.81 & \multicolumn{7}{c}{-33.70} & 10.09 & 7.6 & 0.1 \\
318.15 & 418.59 & 5.777 & -39.49 & 13.14 & 13.2 & 0.1 \\
\hline
\end{tabular}

${ }^{a}$ For $p$ up to $100 \mathrm{MPa}$.

the $\left.P_{\text {int }}\right)$. For 1,3-propanediol, the internal pressure decreases monotonically with increasing pressure along isotherms in every case. At the same time a positive temperature coefficient of internal pressure (at constant pressure) occurs in all investigated diols (see Figs. 1-4).

The maxima of the $P_{\text {int }}$ isotherms for 1,2-butanediol and 1,2-propanediol are shifted towards higher pressures with increasing temperature. In the case of 1,2-butanediol the maximum lies at $23.3 \mathrm{MPa}$, for $T=293.15 \mathrm{~K}$, whereas for $T=$ $313.15 \mathrm{~K}$ it lies at $45.4 \mathrm{MPa}$. In turn, in the case of 1,2-propanediol the maximum 
lies at 7.5 MPa, for $T=293.15 \mathrm{~K}$, whereas for $T=313.15 \mathrm{~K}$ it lies at 27.5 MPa. It should be noted here that a maximum of internal pressure with respect to pressure at constant temperature has been also reported for other liquids [9-13]. Moreover, in contrary to 1-alkanols [10,11], a crossing point of the isotherms not exists for the diols studied. On the other hand, it is very interesting that studied recently 2-methyl-2,4-pentanediol shows such sharp crossing point [14]. It seems that existence of this crossing point can be here related to existence of side chain (methyl group) that restricted (steric hindrance) the formation of intramolecular H-bonds.

The obtained results suggest that relation between internal pressure and ability to form intramolecular hydrogen bonds (five or six atom rings) can exist (obviously all diols are also able to form spatial network of intermolecular $\mathrm{H}$-bonds). This is very interesting because, in the literature the internal pressure is discussed in terms of cohesive forces acting in the liquid and can be interpreted as a measure of non-chemical interactions $[9,15]$. However, the interpretation of the $P_{\text {int }}$ is difficult because it is always possible that $P_{\text {int }}$ includes also contributions from hydrogen bondings. It must be pointed out that although several empirical methods were developed which enable the separation of hydrogen bonding energies from the energies of non-chemical interactions, this separation is still an open problem.

\section{Conclusions}

The ability to form intramolecular hydrogen bond decreases in sequence: 1,3-propanediol, 1,3-butanediol, 1,2-propanediol, and 1,2-butanediol, it seems that this ability can affect the effect of pressure on internal pressure. However, this supposition is rather speculative because it is assumed that internal pressure measures the polar and non-polar (generally non-chemical) interactions within liquid [15]. Thus, from this point of view, internal pressure does not reflect the presence of hydrogen bonding in liquids. In our opinion, however, both hydrogen bond formation as well as non-chemical interactions (structural effects as well) can influence the maximum of internal pressure. Unfortunately, both the present contribution and available literature reports are insufficient to yield any final conclusion.

\section{References}

[1] P. Hemmes, A. Mayevski, V.A. Buckin, A.P. Sarvazyan, J. Phys. Chem. 84, 699 (1980).

[2] E. Zorȩbski, Fortschritte der Akustik DAGA 2002, 724 (2002).

[3] S. Ernst, W. Marczak, R. Manikowski, E. Zorȩbski, M. Zorȩbski, Acoust. Lett. 15, 123 (1992).

[4] E. Zorȩbski, M. Zorȩbski, S. Ernst, in: Ultrasonic World Congress 1995 Proceedings, Humboldt-Universität, Berlin 1995, p. 547.

[5] A. Żak, M. Dzida, M. Zorȩbski, S. Ernst, Rev. Sci. Instrum. 71, 1756 (2000). 
[6] M. Dzida, M. Chorążewski, M. Zorȩbski, S. Ernst, J. Phys. IV (France) 137, 203 (2007).

[7] E. Zorȩbski, M. Dzida, J. Chem. Eng. Data 52, 1010 (2007).

[8] E. Zorȩbski, M. Dzida, M. Piotrowska, J. Chem. Eng. Data 53, 136 (2008).

[9] E. Zorȩbski, Mol. Quant. Acoust. 27, 327 (2006).

[10] E. Zorȩbski, Mol. Quant. Acoust. 28, (2007) in press.

[11] M. Dzida, J. Chem. Eng. Data 52, 521 (2007).

[12] M. Dzida, Eur. Phys. J. Special Topics 154, 271 (2008).

[13] W.J. Moore, Physical Chemistry, 3rd ed., Longmans, London 1959.

[14] E. Zorȩbski, M. Dzida, M. Cempa, Fortschritte der Akustik-DAGA, 2008, in press.

[15] M.R.J. Dack, Chem. Soc. Rev. 4, 211 (1975). 